\title{
Kinetics of Nanoparticle-Membrane Adhesion Mediated by Multivalent Interactions
}

\author{
Roberta Lanfranco,,,$+ \ddagger$ Pritam Kumar Jana, ${ }^{\top}$ Lucia Tunesi ${ }^{\dagger}$ Pietro Cicuta, ${ }^{\dagger}$ \\ Bortolo Matteo Mognetti, "Lorenzo Di Michele, ${ }^{*, \dagger}$ and Gilles Bruylants, ${ }^{*, \dagger}$ \\ $\dagger$ †iological and Soft Systems, Cavendish Laboratory, University of Cambridge, JJ Thomson \\ Avenue, Cambridge CB3 OHE, United Kingdom \\ $\ddagger$ Université libre de Bruxelles (ULB), Engineering of Molecular NanoSystems, 50 av. F.D. \\ Roosevelt, 1050 Brussels, Belgium \\ - Université Libre de Bruxelles (ULB), Interdisciplinary Center for Nonlinear Phenomena \\ and Complex Systems, Campus Plaine, CP 231, Blvd. du Triomphe, B-1050 Brussels, \\ Belgium \\ E-mail: r1578@cam.ac.uk; Id389@cam.ac.uk; gbruylan@ulb.ac.be
}

\section{Abstract}

Multivalent adhesive interactions mediated by a large number of ligands and receptors underpin many biological processes, including cell adhesion and the uptake of particles, viruses, parasites, and nanomedical vectors. In materials science, multivalent interactions between colloidal particles have enabled unprecedented control over the phase behavior of self-assembled materials. Theoretical and experimental studies have pinpointed the relationship between equilibrium states and microscopic system parameters such as ligand-receptor binding strength and their density. In regimes of strong interactions, however, kinetic factors are expected to slow down equilibration, and lead to the emergence of long-lived out-of-equilibrium states that may significantly influence the outcome of self-assembly experiments and the adhesion of particles to biological membranes. Here we experimentally investigate the kinetics of adhesion of nanoparticles to biomimetic lipid membranes. Multivalent interactions are produced by strongly interacting DNA constructs, playing the role of both ligands and receptors. The rate of nanoparticle adhesion is investigated as a function of the surface density of membrane- anchored receptors and of the bulk concentration of nanoparticles, and is observed to decrease substantially in regimes where the number of available receptors is limited compared to the overall number of ligands. We attribute such peculiar behaviour to the rapid sequestration of available receptors after initial nanoparticle adsorption. The experimental trends and the proposed interpretation are supported by numerical simulations.

\section{Introduction}

Adhesive interactions between nanoscale objects and lipid membranes are central for a number of biological processes, including cell-cell communication mediated by extracellular vesicles, ${ }^{1-3}$ viral infection, ${ }^{4-6}$ and endocytosis. ${ }^{7-9}$ In therapeutic and diagnostic nanomedicine, finding reliable strategies to control the interaction between biological membranes and nanoscale probes is a key issue. ${ }^{10-12}$ Adhesion is often mediated by specific ligands on the surface of the nano-objects that target specialised receptors expressed on the cell membranes. The resulting multivalent interactions, mediated by a large number of interacting molecular agents, give 
rise to complex phase behaviours, which emerge from the interplay between enthalpic contributions to individual ligand/receptor interactions and configurational/combinatorial entropic effects. ${ }^{13-18}$ The (bio)physics of multivalent interactions has been thoroughly characterised both experimentally and theoretically, by means of analytical and numerical approaches. ${ }^{15,18-22}$ In the context of materials science, the acquired understanding enabled the design of colloidal or nanoscale units, whose self-assembly behaviour can be precisely prescribed. ${ }^{23-28}$ In targeted drug delivery, multivalent interactions can be exploited to improve the binding selectivity of vectors, which can be designed to target cell membranes only if certain receptors are overexpressed allowing one to discern between healthy and diseased cells. ${ }^{29-32}$

Our current understanding of the equilibrium features of multivalent systems ${ }^{33}$ is not matched by systematic studies of kinetic effects, which are often relevant in regimes where ligand-mediated interactions become sufficiently strong, driving the system into long-lived metastable configurations that differ substantially from the equilibrium ground state. Interactions of such strength can occur in biological recognition schemes, where ligand-receptor binding free energies can exceed $25 k_{\mathrm{B}} T .^{34}$ In multivalent colloidal systems, kinetic arrest has been characterised and exploited to design self-protected interaction schemes or sequential self-assembly protocols. ${ }^{35-39}$ Yet, with the exception of a limited number of studies ${ }^{40-45}$ including the recent investigation of the adhesion kinetics of DNAfunctionalized poly(amidoamine) dendrimers to solid surfaces, ${ }^{46,47}$ systematic quantitative investigations have not been reported on the kinetics of multivalent nanoparticle-surface interactions. Particularly significant and unexplored is the role of kinetic effects in the presence of mobile likers, in these directions will be relevant to the understanding and design of the aforementioned biological and nanomedical processes.

In this paper, we present a quantitative investigation of the adsorption kinetics of nanoparticles to biomimetic lipid membranes. In the adopted model system, Large Unilamellar lipid Vesicles (LUVs) are targeted by gold nanoparticles (NPs), and complementary DNA constructs anchored on both substrates play the role of ligands and receptors, where the latter are able to freely diffuse, as in many biologically relevant situations. ${ }^{15,48-53}$ The programmability and selectivity of Watson-Crick base pairing ensures accurate control over the bond formation, enabling a quantitative characterization of kinetic effects as a function of different parameters characterizing the ligands, the receptors and their complexes. Using Dynamic Light Scattering (DLS), nanoparticle adsorption kinetics is characterized as a function of the surface density of membrane-anchored DNA receptors as well as the overall bulk concentration of nanoparticles. We observe a substantial reduction in adsorption rates in regimes where the number of available receptors is limited compared to the number of nanoparticles. The observed trend is ascribed to the rapid and nearirreversible sequestration of most of the initially available receptors, following the adsorption of a relatively small number of nanoparticles. Receptor depletion is a direct consequence of the mobility of membrane-anchored DNA linkers, and is expected to occur also in biological system, where adhesion is mediated by proteins that can diffuse in the membrane. Experimental evidence is backed up by state-of-the-art simulations that accurately account for the effect of molecular reaction rates on the adsorption dynamics of the nanoparticles.

Our findings demonstrate that, in the presence of strong ligand-receptor interactions, factors such as receptor mobility and concentration should be taken into account when designing nanoscale probes for membrane targeting.

\section{Materials and Experimental Methods}

\section{DNA ligands and receptors}

Single stranded DNA was purchased from IDT (Integrated DNA Technologies, Leuven, Bel- 
gium). Strands mimicking ligands (5' -TGC GTG TGT GCG TTT TTT TTTT-Thiol-3') feature a thiol modification on their $3^{\prime}$ end, enabling grafting to NPs. Receptor strands (5' -56FAM-TCG CAC ACA CGC TTTT TTT TTT-Chol-3') are modified with both a fluorescein $\left(5^{\prime}\right)$ and a cholesterol molecule $\left(3^{\prime}\right)$ (Fig. 1). The latter is connected to the DNA via a TriEthylene Glycol (TEG) spacer, and enables grafting onto lipid bilayers. Ligand and Receptor strands feature mutually complementary sticky ends of 11 nucleotides, driving their hybridization and NP-membrane adhesion. PolyT domains are included between the sticky overhangs and the grafting moieties to improve configurational freedom.

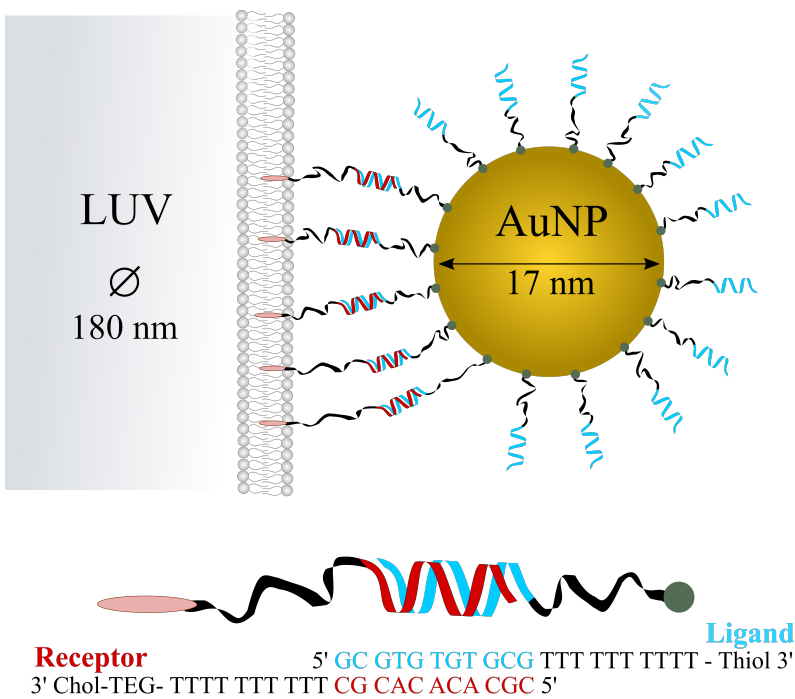

Figure 1: Hybridization between DNA ligands and receptors drives the adhesion of a NP to a lipid membrane. The sequences of ligand and receptor DNA strands are reported. They feature, respectively, a thiol and a TEG-cholesterol moiety to anchor them to NPs and LUVs.

\section{Liposome preparation, functional- ization, and characterization}

Large Unilamellar Vesicles (LUVs) were prepared from 1-palmitoyl-2-oleoyl-glycero-3phosphocholine (POPC) lipids (Avanti Polar Lipids, CAS number 26853-31-6) by membrane extrusion, using an Avanti mini-extruder. First, a dry lipid film is obtained by evaporation of a chloroform solution under vacuum for 1 hour. The lipid film is then rehydrated with experimental buffer $(10 \mathrm{mM}$ phosphate buffer pH 7.4 $+0.5 \mathrm{M} \mathrm{NaCl}$ ). Rehydration is facilitated by vortexing the sample for $1 \mathrm{~min}$ and further incubating for 1 hour. The solution is then extruded 31 times through a poly-carbonate membrane featuring track-etched pores with a diameter of $200 \mathrm{~nm}$ (Whatman). A high number of extrusion steps is necessary to obtain a homogeneous size distribution of the liposomes. As prescribed by the manufacturer of the extrusion kit (Avanti Polar Lipids), an odd number of steps is required so that any particulate contaminant larger than the pore size is blocked by the membrane and removed from the sample. The formed liposomes have a hydrodynamic diameter, measured with DLS (Malvern Zetasizer NanoZS, Malvern, UK), of either $160 \pm 31 \mathrm{~nm}$ or $189 \pm 37 \mathrm{~nm}$ (SI, section 1.1). The overall lipid concentration $(2 \mathrm{mM})$ and the hydrodynamic diameter were used to estimate the bulk concentration of the liposomes. Vesicles were functionalized with different concentrations of Receptor strands, to obtain surface densities in a biologically relevant range ${ }^{54,55}$ (SI, section 1.2). Functionalization was performed by adding DNA Receptors to the LUV solution and agitating on an Eppendorf thermomixer for 16 hours at $750 \mathrm{rpm}$. The cholesterol moiety spontaneously inserts into the lipid bilayer, providing a mobile but stable anchoring. ${ }^{56-58}$ To demonstrate the grafting of all DNA strands to the LUVs, samples were analysed by Ultra Centrifugation (Optima LE-80K from Beckman) in a sucrose gradient (SI, section 1.3).

\section{NPs synthesis, functionalization, and characterization}

The materials used to synthesize NPs: Potassium gold (III) tetrachloride $\left(\mathrm{KAuCl}_{4}\right.$, CAS number 450235), dithiothreitol (DTT, CAS number 3483-12-3), trisodium citrate $\left(\mathrm{Na}_{3} \mathrm{C}_{6} \mathrm{H}_{5} \mathrm{O}_{7}\right.$, CAS number 51 804), and Tris$\mathrm{HCl}$ buffer (1 M, pH 7, CAS number T6455), were purchased from Sigma-Aldrich, while 
phosphate buffer and $\mathrm{NaCl}$ used for particle functionalization were purchased from Merck. Gold nanoparticles (NPs) were produced via a modified Turkevich method ${ }^{59}$ and immediately dialyzed against a $0.1 \mathrm{mM}$ citrate solution. The nanoparticles were characterized by TEM, UVvis absorption, and DLS measurements, as reported in SI, section 2. An average diameter of $16.5 \pm 1.3 \mathrm{~nm}$ was determined by processing TEM images with ImageJ. NPs were then functionalized with DNA Ligands using thiol chemistry. ${ }^{60}$ The grafting density was estimated by measuring the DNA concentration of the supernatant after a 20-minute centrifugation step at $18,000 \mathrm{~g}$. The concentration of DNA in the supernatants after the first washing step was measured as $2.24 \mu \mathrm{M}$, while the initial DNA concentration was $3.23 \mu \mathrm{M}$, demonstrating the occurrence of DNA grafting. Afterwards, the same washing procedure was repeated 5 times to remove all the non-grafted DNA. We obtained an average of 300 Ligand strands per NP. The presence of a DNA layer on NPs was confirmed by both DLS and UV-vis, as reported in SI, section 2.

\section{Characterizing NP-LUV adhesion and dissociation temperature.}

The adhesion of NPs to LUVs was confirmed by means of UV-vis absorption spectroscopy (Schimadzu UV-3600 equipped with a Peltier T controller) monitoring the shift in the wavelength of the NP extinction peak induced by the reciprocal proximity between LUV-bound NPs. The dissociation temperature of the NP-LUV complexes was also determined and found to increase with increasing surface density of the receptors $\rho_{\mathrm{R}-\mathrm{LUV}}$. Details are provided in SI, section 3 .

\section{Characterizing NP-LUV adhesion with Dynamic Light Scattering}

Experiments to monitor the adhesion kinetics of NPs to LUVs were carried out using Dynamic Light Scattering (DLS). Initially, a sample of DNA-functionalized NPs in $0.5 \mathrm{M}$
$\mathrm{NaCl}+10 \mathrm{mM}$ phosphate buffer at concentrations reported in the paragraph List of Samples, tipically in the range of $0.1-0.2 \mathrm{nM}$, was characterized via DLS. The scattering autocorrelation function (ACF) was used to quantify the diffusion parameters of free NPs, discussed in the Results and Discussion section. Then, LUVs were added to reach the sought NPs:LUVs bulk concentration ratio $R_{\mathrm{NP}: L U V}$, and the sample was rapidly mixed with a pipette. DLS data acquisition started typically $10 \mathrm{~s}$ after mixing, and 60 ACFs were acquired at $5 \mathrm{~s}$ intervals. The time interval between subsequent measurements was then increased to 30 seconds, 3 minutes, and $15 \mathrm{~min}$ utes to monitor adhesion over the following $14 \mathrm{~h}$. All measurements were performed at $25^{\circ} \mathrm{C}$ and in $0.5 \mathrm{M} \mathrm{NaCl}$ ionic strength. Higherthan-physiological ionic strength of $0.5 \mathrm{M} \mathrm{NaCl}$ was used in this study to obtain sufficiently high ligand-receptor binding strength to allow the emergence of significant kinetic effects associated to the near-irreversibility of the NP adhesion to the target vesicles. ${ }^{61}$ The effect of a higher-than-physiological ionic strength is largely limited to the DNA-hybridization free energy, and therefore our results are readily applicable to any system with comparably strong ligand-receptor interactions, regardless of the ionic strength. Similar ionic conditions are routinely used in DNA-nanosystems to make DNA duplexes more stables. ${ }^{62-65}$

\section{List of samples}

A number of samples were tested with different surface concentration of receptors on the LUVs, $\rho_{\mathrm{R}-\mathrm{LUV}}$, spanning a biologically relevant range, ${ }^{54,55}$ and different NP:LUV bulk concentration ratios, $R_{\mathrm{NP}: \mathrm{LUV}}$. All tested conditions are listed in Table 1 . For samples with a $R_{\mathrm{NP}: \mathrm{LUV}}=7$ and 70 , the bulk nanoparticles concentration $\rho_{N P}$ is $1.1 \times$ $10^{-10} \mathrm{M}$, while $R_{\mathrm{NP}: \mathrm{LUV}}=290$ corresponds to $\rho_{N P}=2.2 \times 10^{-10} \mathrm{M}$. 
Table 1: List of tested samples: $R_{\mathrm{NP} \text { LUV }}$ indicates the NP:LUV bulk concentration ratio, $\rho_{\mathrm{R}-\mathrm{LUV}}$ the average surface concentration of receptors on the LUVs, $N_{\mathrm{R}-\mathrm{LUV}}$ the average total number of receptors on each LUV, and $N_{\mathrm{R}-\mathrm{NP}}=N_{\mathrm{R}-\mathrm{LUV}} / R_{\mathrm{NP}: \text { LUV }}$ the average number of Receptors available for each NP in the system. The number of ligands on each nanoparticle is fixed and equal to 300, see SI, section 1.2 for details on the estimation of these values.

\begin{tabular}{c|c|c|c|c} 
Sample & $R_{\text {NP:LUV }}$ & $\rho_{\text {R-LUV }}\left(\mathrm{nm}^{-2}\right)$ & $N_{\text {R-LUV }}$ & $N_{\text {R-NP }}$ \\
\hline 1 & 7 & $3.910^{-3}$ & 370 & 53 \\
2 & 7 & $8.210^{-3}$ & 770 & 110 \\
3 & 7 & $12.710^{-3}$ & 1190 & 170 \\
4 & 7 & $17.510^{-3}$ & 1640 & 234 \\
5 & 70 & $3.910^{-3}$ & 370 & 5 \\
6 & 70 & $8.210^{-3}$ & 770 & 11 \\
7 & 70 & $12.710^{-3}$ & 1190 & 17 \\
8 & 70 & $17.510^{-3}$ & 1640 & 23 \\
9 & 290 & $3.910^{-3}$ & 370 & 1 \\
10 & 290 & $8.210^{-3}$ & 770 & 3 \\
11 & 290 & $12.710^{-3}$ & 1190 & 4 \\
12 & 290 & $17.510^{-3}$ & 1640 & 6
\end{tabular}

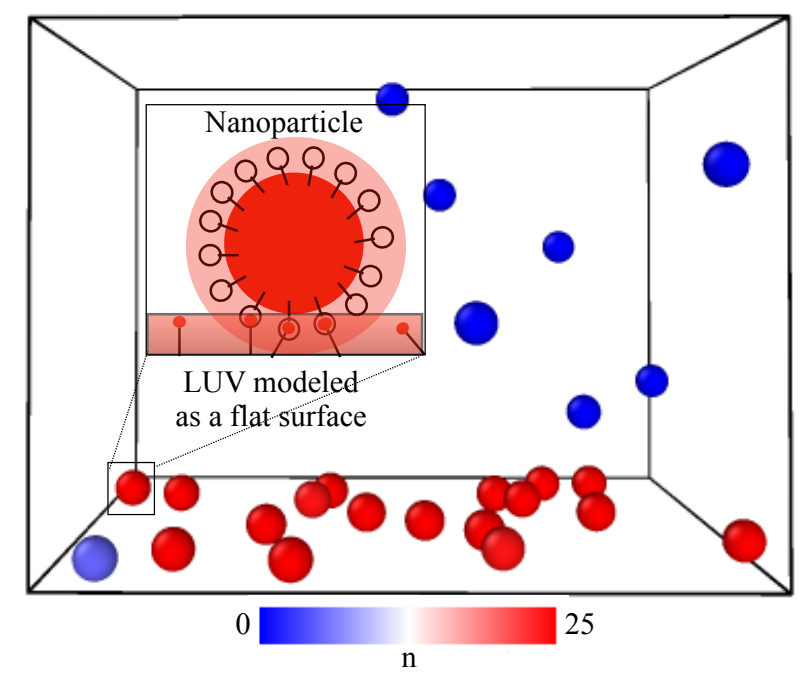

Figure 2: Modelling ligand-receptor mediated interactions between NPs and membranes. Ligand and receptor sticky-ends are uniformly distributed, respectively, in the blue and red stained regions surrounding the NP and the membrane surface. Inset: simulation snapshot showing NPs anchored at the surface or freely diffusing in bulk. The colormap represents the number of formed NP-membrane bonds.

\section{Simulation Algorithm}

We model NPs as hard spheres freely diffusing in a parallelepipedal simulation box (Fig. 2). Nanoparticles interact with the basal surface of the simulation box having area $A$, equal to that of a LUV. The surface carries $N_{\mathrm{R}-\mathrm{LUV}}$ receptors, a number compatible with the one used in experiments. As often done when modelling DNA-functionalized surfaces,${ }^{16,18}$ we neglect receptor-receptor, receptor-ligand, and ligand-ligand steric interactions. We assume that the sticky ends tethered to the surface of the LUV are uniformly distributed and capable of free diffusion within a layer of thickness $\ell=3 \mathrm{~nm}$ surrounding the surface of the LUV (see Fig. 2). $\ell$ has been estimated using typical end-to-end distances of ssDNA. ${ }^{66} \mathrm{Sim}-$ ilarly, nucleic acids on the NPs are simulated as $N_{\mathrm{L}-\mathrm{NP}}$ sticky-ends uniformly distributed within a shell of thickness $\ell$ surrounding the particle (see Fig. 2). Given that in our modelling all ligands can bind, $N_{\mathrm{L}-\mathrm{NP}}$ has been estimated by calculating the maximum number of ligands that could bind the surface using a geometrical construction (see SI, section 4). To improve computational efficiency, also the ligands are regarded as freely diffusive, rather than anchored to a point on the NP surface. This approximation has a negligible effect on the results of this work. We apply the simulation algorithm presented in reference ${ }^{67}$ to study DNA-directed self-assembly of LUVs, adapted to the present system. Improving on conventional algorithms, our method synchronizes the binding kinetics of DNA with the diffusion kinetics of the nanoparticles, enabling one to study the effects of finite interaction rates on the adsorption kinetics. Note that our model does not account for the deformability of the LUVs. While membrane deformability is certainly pivotal in processes such as endocytosis ${ }^{68-73}$ or membrane--mediated interaction between bound particles, ${ }^{74,75}$ it is expected to play little role in the present study to the extent that reactions between different ligand-receptor pairs can be considered as independent events. We have verified this hypothesis in a recent publication ${ }^{19}$ using a more detailed model account- 
ing for membrane deformability. We also notice that an eventual wrapping of the NPs by the lipid bilayer would not change the conclusions of the paper. Indeed, here we describe how receptors, once bound, no longer participate in the later stages of the adsorption. This scenario is not affected by the NPs being wrapped or not.

Nanoparticle positions $\left(\mathbf{r}_{i}\right)$ are updated $\left(\mathbf{r}_{i} \rightarrow\right.$ $\mathbf{r}_{i}+\Delta \mathbf{r}_{i}$ ) using a Brownian dynamics scheme

$$
\begin{gathered}
\Delta \mathbf{r}_{i}=\mathbf{r}_{i}(t+\Delta t)-\mathbf{r}_{i}(t)= \\
\mathbf{f}_{i} \frac{D}{k_{\mathrm{B}} T} \Delta t+\sqrt{D \Delta t} \mathcal{N}(0,1),
\end{gathered}
$$

where $\mathbf{f}_{i}$ is the total force acting on NP $i$, $D$ is the nanopaticle bulk diffusion coefficient in diluted conditions (estimated using StokesEinstein equation as $\left.2.8 \times 10^{7} \mathrm{~nm}^{2} \mathrm{~s}^{-1}\right), \Delta t$ is the integration step, $\mathcal{N}$ is a vector of independent normal-distributed numbers, $k_{\mathrm{B}}$ is the Boltzmann constant and the index $i$ labels individual NPs. In the (very diluted) bulk (see Fig. 2), nanoparticles repel each other through a short-range force $\mathbf{f}_{i j}$ due to entropic compression of grafted DNA. ${ }^{18} \mathbf{f}_{i}$ (Eq. 1) is then given by $\mathbf{f}=\mathbf{f}_{\mathrm{R}, i}+\sum_{j} \mathbf{f}_{i j}$, where $\mathbf{f}_{\mathrm{R}, i}$ is the force between the surface and NP $i$

$\mathbf{f}_{\mathrm{R}, i}=k_{\mathrm{B}} T\left[n_{i}^{\mathrm{s}} \Gamma_{1}(\{\mathbf{r}\})-n^{\mathrm{s}} \Gamma_{2}(\{\mathbf{r}\})-n_{i} \Gamma_{3}(\{\mathbf{r}\})\right]$.

In Eq. $2, n_{i}^{\mathrm{s}}$ is the number of ligand-receptor bonds between nanoparticle $i$ and the membrane, $n^{\mathrm{s}}$ is the number of free receptors, and $n_{i}$ the number of free ligands on particle $i . \Gamma_{1}$, $\Gamma_{2}$ and $\Gamma_{3}$ are geometric factors and have been derived in SI, section 4.

We estimate $n_{i}^{\mathrm{s}}, n^{\mathrm{s}}$, and $n_{i}$ using the Gillespie method. ${ }^{76}$ At a given configuration $\{\mathbf{r}\}$, we calculate all the on/off rates $\left(k_{\text {on }}^{i} / k_{\text {off }}^{i}\right)$ of forming/breaking a bond between nanoparticle $i$ and the membrane, with the assumption that $k_{\mathrm{on}}^{i}=k_{\mathrm{off}}^{i}=0$ if nanoparticle $i$ is not in contact with the interface. In the SI, section 4 , we prove that ${ }^{39}$

$$
k_{\text {on }}^{i}=k_{\text {on }}^{0} \lambda_{i}(\{\mathbf{r}\}) \quad k_{\text {off }}^{i}=k_{\text {on }}^{0} \rho_{0} \exp \left[\beta \Delta G_{0}\right],
$$

where $\lambda_{i}(\{\mathbf{r}\})$ are geometric factors (see SI, section 4), $\Delta G_{0}$ is the hybridization free energy of free sticky ends in solution, ${ }^{61} \beta=1 / k_{\mathrm{B}} T, k_{\mathrm{on}}^{0}$ is the on rate of the sticky ends, and $\rho_{0}$ is the standard concentration equals to 1 mole per liter. In the following we report on rates in simulation units: $k_{\text {on }}^{0, *}=k_{\text {on }}^{0} \rho_{0} \ell^{2} D^{-1}$. In physical units, $k_{\text {on }}^{0, *}=1$ corresponds to $k_{\text {on }}^{0}=3.1 \times 10^{6} \rho_{0}^{-1} \mathrm{~s}^{-1}$. Using the rates of Eq. 3 we calculate the probabilities $p_{\text {on }}^{i} / p_{\text {off }}^{i}$ of forming / breaking a bond between nanoparticle $i$ and the membrane, defined as

$$
\begin{array}{cc}
p_{\mathrm{on}}^{i}=\frac{a_{\mathrm{on}}^{i}}{a_{\mathrm{tot}}}, & p_{\mathrm{off}}^{i}=\frac{a_{\mathrm{off}}^{i}}{a_{\mathrm{tot}}} \\
a_{\mathrm{on}}^{i}=n_{i} n^{\mathrm{s}} k_{\mathrm{on}}^{i} & a_{\mathrm{off}}^{i}=n_{i}^{\mathrm{s}} k_{\mathrm{off}}^{i},
\end{array}
$$

where $a_{\text {on }}^{i}$ and $a_{\text {off }}^{i}$ are the corresponding affinities and $a_{\text {tot }}=\sum_{i=1}^{N_{p}}\left(a_{\mathrm{on}}^{i}+a_{\text {off }}^{i}\right)$, where $N_{p}$ is the total number of nanoparticles in the simulation box. Using Eq.4, we sample one of all possible reactions, along with the average time $(\bar{\tau})$ for it to happen $\left(\operatorname{Prob}(\bar{\tau}) \sim \exp \left[-\bar{\tau} / a_{\text {tot }}\right]\right)$. We increment a reaction clock $\left(\tau_{\text {reac }}\right)$ by $\bar{\tau}\left(\tau_{\text {reac }}=\right.$ $\left.\tau_{\text {reac }}+\bar{\tau}\right)$ and, if $\tau_{\text {reac }}<\Delta t$, we update the values of $\left\{n_{i}, n_{i}^{\mathrm{s}}, n^{\mathrm{s}}\right\}$, the affinities and probabilities (Eqs. 5, 4), and fire another reaction until $\tau_{\text {reac }}>\Delta t$. Importantly, the last reaction is never used to update the values of $\left\{n_{i}, n_{i}^{\mathrm{s}}, n^{\mathrm{s}}\right\}$. Using the outcomes of the Gillespie algorithm at time $\Delta t$, we calculate forces using Eq. 2 and update nanoparticle positions using Eq.1. At which point, we re-iterate the algorithm starting from the calculation of the new on/off rates (Eq. 3).

To simulate the low dilution regimes used in experiments, we use grand-canonical moves in which we insert/delete nanoparticles at the top layer of the simulation box. To simulate a fixed NP:LUV concentration ratio, we gradually decrease the chemical potential $\mu$ of the NPs as more of them are adsorbed by the membrane using $\mu=\mu_{0}+\log \left(1-n_{\mathrm{adh}} / R_{\mathrm{NP}: \mathrm{LUV}}\right)$, where $\mu_{0}$, $n_{\text {adh }}$, and $R_{\mathrm{NP}: \mathrm{LUV}}$ are, respectively, a reference chemical potential, the number of adsorbed particles, and the stoichiometric ratio between NPs and LUVs. Grand-canonical moves are necessary to design affordable simulations but intro- 

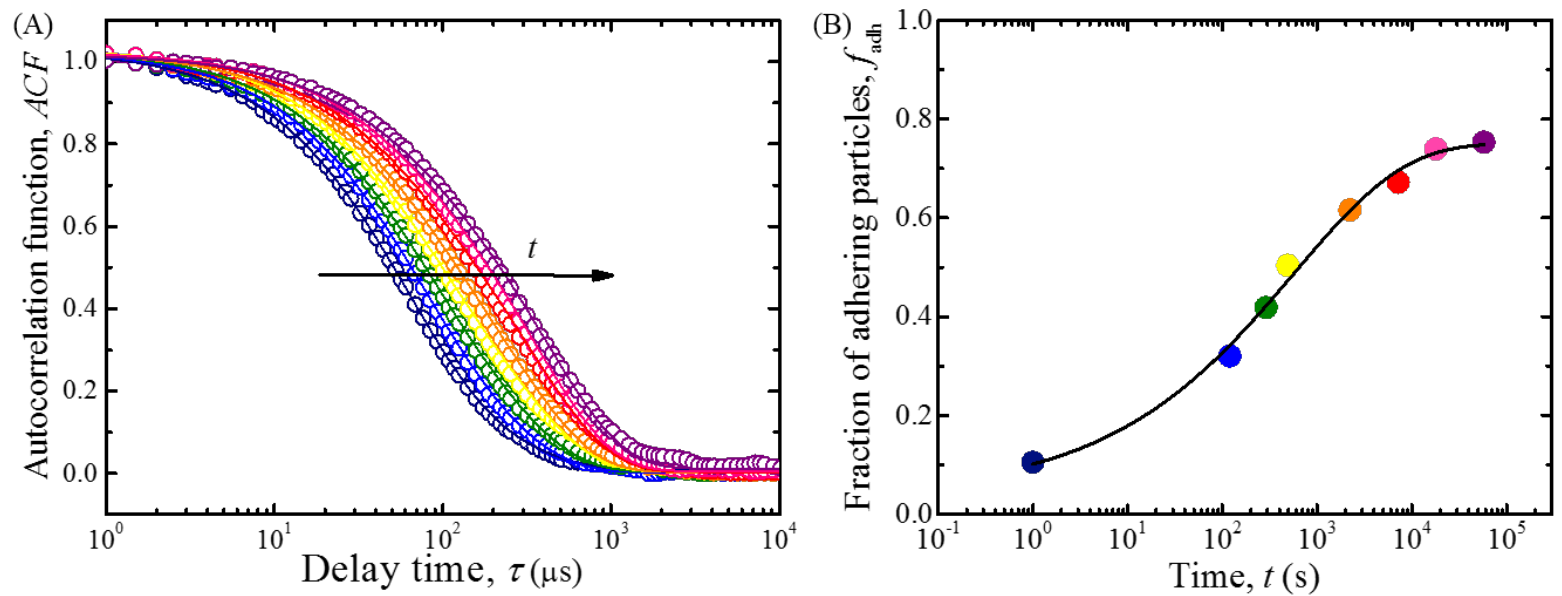

Figure 3: Quantifying adhesion kinetics with DLS. A) Autocorrelation functions acquired with DLS for a system with $R_{\mathrm{NP}: \mathrm{LUV}}=70$ and $\rho_{\mathrm{R}-\mathrm{LUV}}=17.5 \times 10^{-3} \mathrm{~nm}^{-2}$. The ACF was collected after different times $t$ at the mixing LUVs and NPs (dark blue $t=0 \mathrm{~s}$, light blue $t=120 \mathrm{~s}$, green $t=290 \mathrm{~s}$, yellow $t=490 \mathrm{~s}$, orange $t=2220 \mathrm{~s}$, red $t=7200 \mathrm{~s}$, pink $t=18000 \mathrm{~s}$ and purple $t=57600 \mathrm{~s}$.) B) Fraction of bound NPs, $f_{\mathrm{adh}}$, is obtained by fitting the data from panel A with Eq. 6, extracting the two amplitudes $A_{\text {free }}(t)$ and $A_{\text {adh }}(t)$, and using Eq. 7. The color code is as in panel A. The fraction of adhering nanoparticles, $f_{\mathrm{adh}}$, for all the perfomed experiments are reported in SI, section 9, Fig S9.1. Notice that only a subset of the collected ACFs are reported in panel A.

duce a bias in the diffusion time required by nanoparticles to reach the membrane, making it significantly faster than in reality.

\section{Results and Discussion}

We use DLS to investigate kinetic aspects of the interaction between NPs and LUVs decorated by DNA ligands and receptors (Fig. 1) as a function of receptor surface density on the LUVs, $\rho_{\mathrm{R}-\mathrm{LUV}}$, and NP:LUV stoichiometric ratio, $R_{\mathrm{NP}: \mathrm{LUV}}$, spanning the ranges described in Table 1. In DLS experiments, the signal is dominated by the strongly scattering NPs, while owing to the similarity between the refractive index of the vesicles and that of the surrounding medium, the scattering contribution of the LUVs is comparatively negligible. (see SI, section 5). When an individual NP adheres to a liposome, whose size is approximately one order of magnitude larger, the dynamic diffusion coefficient of the nanoparticle is drastically reduced. After NPs are exposed to LUVs, this effect produces a progressive shift to longer decay times of the scattering autocorrelation function $(\mathrm{ACF})$, as demonstrated in Fig. 3A, which can thus be monitored to gain information on nanoparticle adhesion. For any measurement, we can identify two distinct NP populations: free nanoparticles and nanoparticles bound to the LUVs. Over time, the population of free NPs progressively shrinks, while the bound population grows. Therefore the measured ACF can be decomposed into the sum of two exponentials, one describing the free NPs population and the second associated to membrane-bound NPs

$$
\operatorname{ACF}(\mathrm{t}, \tau)=A_{\text {free }}(t) e^{-\left(2 \tau / \tau_{\text {free }}\right)^{\alpha}}+A_{\text {adh }}(t) e^{-2 \tau / \tau_{\text {comp }}},
$$

where $t$ is the time elapsed from the beginning of the experiment, and $\tau$ indicates the delay time over which each scattering-intensity correlation is calculated. The timescales $\tau_{\text {free }}$ and $\tau_{\text {comp }}$ are related to the diffusion coefficient of the free nanoparticles and the NP-LUV complexes, respectively, while $\alpha<1$ follows from the polydispersity of the NP population. ${ }^{77}$ Note that using a stretched exponential to model the 

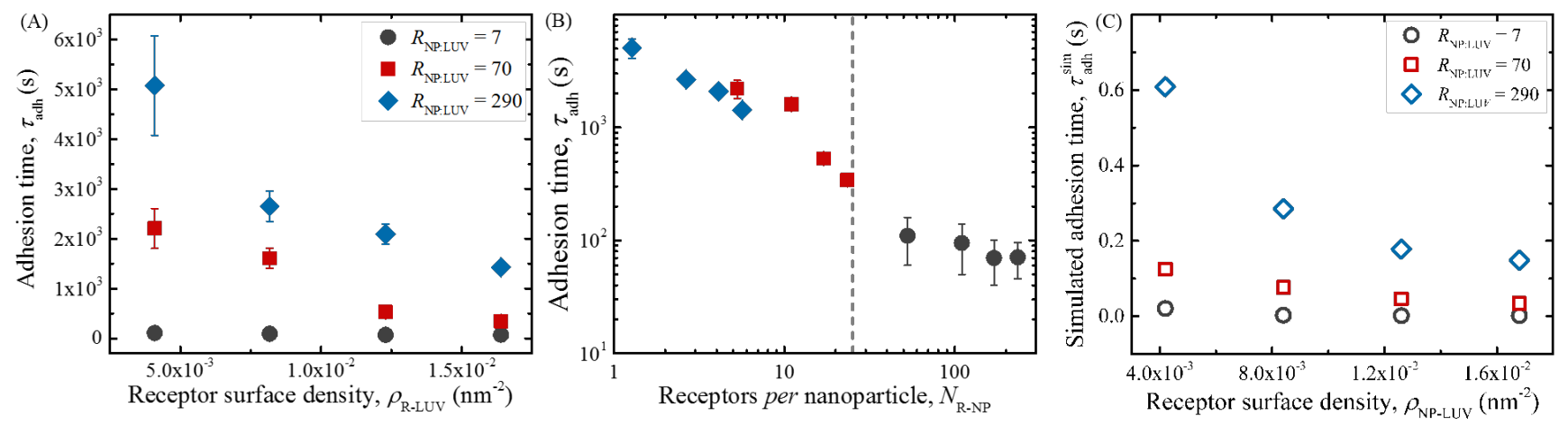

Figure 4: Adhesion kinetics depends on receptor density and nanoparticle concentration. A) Adhesion times $\tau_{\text {adh }}$ for three different NP:LUV concentration ratios $R_{\mathrm{NP}: \mathrm{LUV}}$ as a function of the receptor surface density $\rho_{\mathrm{R}-\mathrm{LUV}}$. B) The same data as in panel A, shown as a function of the average number of receptors per NP in the system $N_{\mathrm{R}-\mathrm{NP}}$. The vertical dashed line marks the maximum number of bonds a nanoparticle can form due to geometrical limitations $N_{\text {bonds }}^{\max }=25$ (see SI, section 7.1). C) Simulated adhesion times $\tau_{\text {adh }}^{\text {sim }}$, using $\tau_{\text {adh }}^{\text {sim }}=-\Delta t / \log (1-f(\Delta t))$, where $f(\Delta t)$ is the fraction of nanoparticles adsorbed at time $\Delta t$, with $\Delta t$ small enough to allow fitting data with an exponential function. The predictions of panel A are bigger than the values of panel B because the average time between consecutive NP-LUV encounter is smaller in simulations. Nevertheless, the similarity between the two figures confirms that the reaction kinetics is limiting adsorption.

contribution of the complexes did not improve the fits, and the stretching factor was always found to be comparable to 1 . In Eq. 6, the amplitudes $A_{\text {free }}(t)$ and $A_{\text {adh }}(t)$ are directly proportional to the number of free and adhering nanoparticles, which allows us to extract the fraction of bound nanoparticles as

$$
f_{\mathrm{adh}}(t)=\frac{A_{\mathrm{adh}}(t)}{\left[A_{\mathrm{adh}}(t)+A_{\mathrm{free}}(t)\right]} .
$$

Eq. 7 is valid under the assumption that free and membrane-bound NPs have the same scattering efficiency. This assumption is motivated and discussed in SI, section 6 . Figure 3B demonstrates how $f_{\text {adh }}$ increases with time as a stretched exponential

$$
f_{\mathrm{adh}}(t)=f_{\mathrm{adh}}^{\mathrm{ss}}\left[1-\exp \left(-\frac{t}{\tau_{\mathrm{adh}}}\right)^{\gamma}\right]
$$

where $\tau_{\text {adh }}$ quantifies the typical adhesion timescales, and the plateau value $f_{\mathrm{adh}}^{\mathrm{ss}}$ represents the steady state fraction of adsorbed nanoparticles. Optimal fitting is obtained by restricting the stretching factor $\gamma$ in the range of $0.9-1,0.6-0.7$ and $0.4-0.5$ for $R_{\mathrm{NP}: \mathrm{LUV}}=$
7,70, and 290, respectively. The stretchedexponential trend hints at a hindered adsorption dynamics in which the rate of NP adhesion decreases over time. Similar trends have been ascribed to a number of processes including depletion of adsorbing agents, ${ }^{78,79}$ steric hindrance and irreversible adsorption. ${ }^{80}$ The progressive decrease of $\gamma$ with increasing $R_{\mathrm{NP}: L U V}$ we observed indicates that the specific factor limiting adsorption in our system becomes more severe as the number of available nanoparticles increases. Note that in Eq. 6 the parameters $\tau_{\text {free }}$ and $\alpha$ describe intrinsic characteristics of NPs that do not change over time, and can therefore be independently determined and kept constant. The decay time $\tau_{\text {comp }}$ describes the diffusion of NP-LUV complexes and evolution over the course of an experiment is analysed in a dedicated section below.

\section{Reaction-limited nanoparticle ad- hesion and receptor depletion}

Figure 4A demonstrates the dependence of the adhesion time $\tau_{\text {adh }}$ on the NP:LUV rela- 

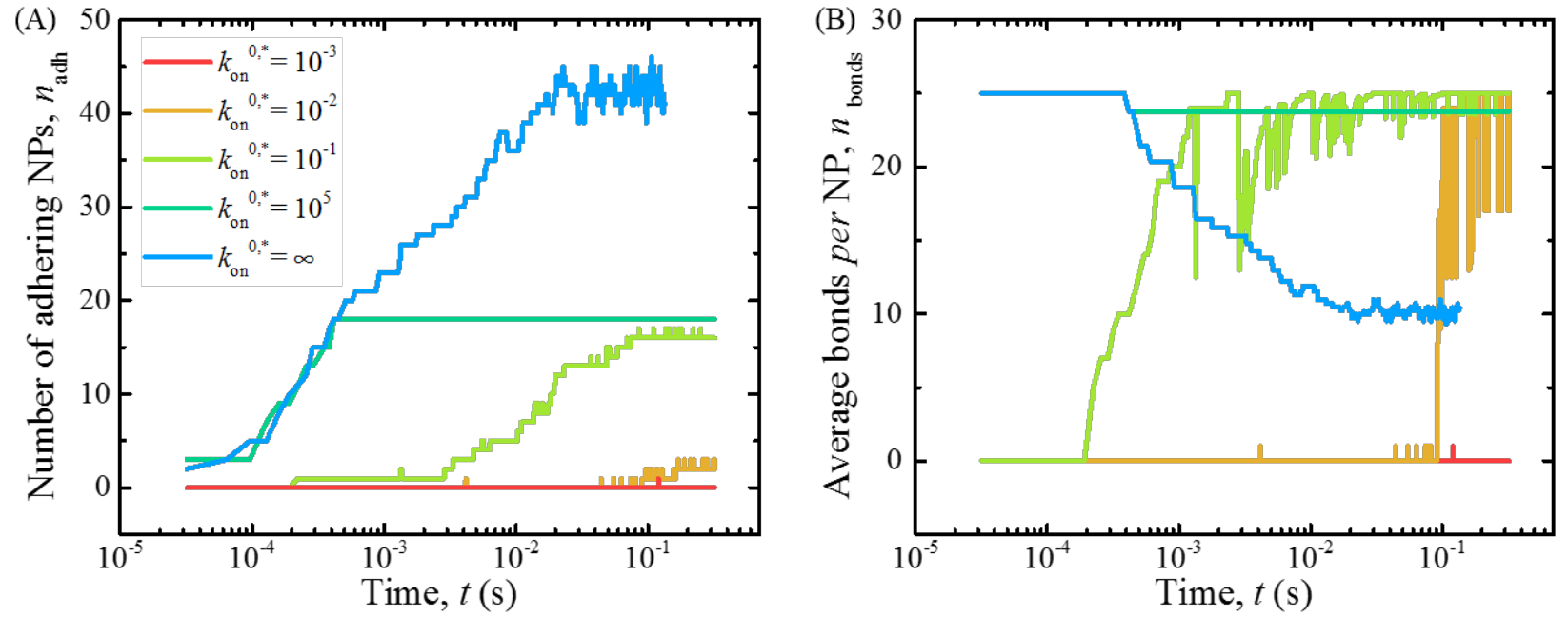

Figure 5: Simulations highlight the effect of kinetic rates on particle adhesion. A) Number of adhering nanoparticles $n_{\text {adh }}$ as a function of time for different reaction rates parametrized by $k_{\text {on }}^{0, *}$ (see sections on Simulation Algorithm). When $k_{\mathrm{on}}^{0, *}<\infty$ the system fails to reach the equilibrium state corresponding to the steady value of the $k_{\mathrm{on}}^{0, *}=\infty$ trajectory. Receptors surface density was set to $\rho_{\mathrm{R}-\mathrm{LUV}}=4.2 \times 10^{-3} \mathrm{~nm}^{-2}$ and the NP:LUV concentration ratio to $R_{\mathrm{NP}: \mathrm{LUV}}=70$. B) Average number of bonds $n_{\text {bonds }}$ formed by each adhering nanoparticle for the simulated trajectories of panel A.

tive concentration ratio $R_{\mathrm{NP}: \mathrm{LUV}}$ and the surface density of DNA receptors on the LUVs $\rho_{\mathrm{R}-\mathrm{LUV}}$. For fixed $\rho_{\mathrm{R}-\mathrm{LUV}}, \tau_{\mathrm{adh}}$ is observed to increase with increasing $R_{\mathrm{NP}: \mathrm{LUV}}$. If the latter is kept fixed, $\tau_{\text {adh }}$ decreases monotonically with increasing $\rho_{\mathrm{R}-\mathrm{LUV}}$. The samples with the lowest $R_{\mathrm{NP}: \mathrm{LUV}}$, where $\tau_{\mathrm{adh}}$ remains constant, constitute an exception. These trends can be better rationalized by studying the dependence of $\tau_{\mathrm{adh}}$ on the number $N_{\mathrm{R}-\mathrm{NP}}$ of receptors per NP, obtained by dividing the average number $N_{\mathrm{R}-\mathrm{LUV}}$ of receptors anchored on each LUV by $R_{\mathrm{NP} \text { LUV }}$ (Table 1 ). The data in Fig. $4 \mathrm{~B}$ show indeed a smooth trend, and clearly highlight two distinct regimes: $\tau_{\text {adh }}$ is low and constant for high $N_{\mathrm{R}-\mathrm{NP}}$, while increasing monotonically as $N_{\mathrm{R}-\mathrm{NP}}$ decreases below a threshold. The switching point coincides with $N_{\mathrm{R}-\mathrm{NP}} \sim 20-50$, which matches well the maximum number of ligand-receptor bonds that a single NP can form due to geometrical limitations, estimated as $N_{\text {bonds }}^{\max } \sim 25$ (see SI, section $7.1)$.

A qualitative explanation of the observed ki- netic trends can be proposed by comparing the timescales of the individual processes leading to NP adhesion. Adsorption is initiated by a collision between a NP and a LUV, and the average time $\tau_{\text {diff }}$ between two of these events, at the beginning of the experiment, ranges between 80 and $450 \mathrm{~ms}$ (see SI, section 8.1). However, during each collision, the NP-LUV spend a much shorter time within their interaction range, $\tau_{\text {coll }} \approx \ell^{2} / D=0.3 \mu \mathrm{s}$. While a NP is in contact with a LUV, the time required for the formation of a ligand-receptor bond is $\tau_{\text {bond }} \lesssim 200 \mathrm{~ms}$ (see Eq.3 and SI, section 8.2), indicating that several thousands of unsuccessful collisions $n_{\text {att }}$ $\left(n_{\text {att }} \approx \tau_{\text {bond }} / \tau_{\text {coll }}\right)$ are on average required for a NP to dock on a LUV. Assuming a collision rate of the order of $\sim \tau_{\text {diff }}^{-1}, \tau_{\text {diff }}<0.5 \mathrm{~s}$, the typical timescale at which particles start adsorbing can then be estimated using $n_{\text {att }} \cdot \tau_{\text {diff }} \approx 10^{3}$ $10^{4} \mathrm{~s}$ which is compatible with Fig. $4 \mathrm{~A}$. After the first bond is established, preventing the LUV and NP from diffusing apart, more connections are quickly formed as mobile receptors are converging towards the adhesion zone. Receptor accumulation occurs on the timescale 

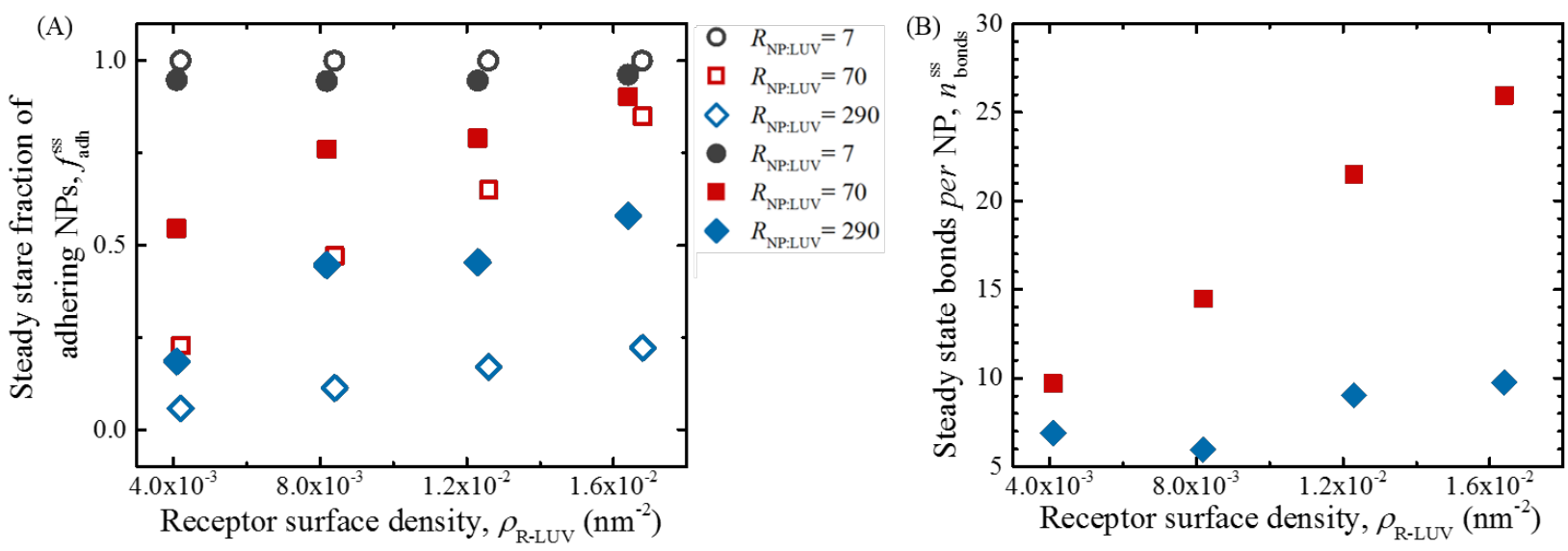

Figure 6: Receptor availability influences steady state configuration. A) Steady state fraction of adhering NPs $f_{\text {adh }}^{s s}$ as estimated from experiments (full symbols) and simulations (empty symbols) as a function of $R_{\mathrm{NP}: \mathrm{LUV}}$ and $\rho_{\mathrm{R}-\mathrm{LUV}}$. B) Experimental estimate of the steady-state average number of bonds per adhering NP $n_{\text {bonds }}^{\text {ss }}$

$\tau_{\text {bond }} \ll n_{\text {att }} \cdot \tau_{\text {diff }}$, so that before another NP has a chance to bind with the LUV, the first has already maximised the number of bonds, capturing $N_{\text {bonds }}^{\max }$ receptors. If $N_{\mathrm{R}-\mathrm{NP}}$ exceeds $N_{\text {bonds }}^{\max }$, the process continues until all nanoparticles have formed $N_{\text {bonds }}^{\max }$ connections, leaving free receptors on the LUVs. In turn, for lower $N_{\mathrm{R}-\mathrm{NP}}$, the system enters a regime where the initially binding NPs quickly deplete receptors, drastically reducing their availability. Receptor scarcity causes $\tau_{\text {bond }}$ to increase, reducing the chances of a successful docking and substantially slowing down adhesion. In our experiments, receptor depletion is expected to be effectively irreversible due to the strong affinity between DNA ligands and receptors resulting in a bond breakup rate $k_{\text {off }}^{\mathrm{i}} \sim 10^{6} \exp \left(\beta \Delta G_{0}\right)$ $s^{-1}$ (see Eq. 3). The initial transient, therefore, drives the system out of equilibrium. At high NP:LUV concentration ratio $-R_{\mathrm{NP}: \mathrm{LUV}}=290$ - steric hindrance can further limit nanoparticle adsorption at later stages given that at full packing a LUV can host a maximum of $\sim 150$ NP (SI, section 7.2).

These qualitative arguments can be verified by comparing experimental data with simulated trends. Figure $4 \mathrm{C}$ reports adhesion timescales $\tau_{\text {adh }}^{\text {sim }}$, estimated by fitting the early stages of adsorption as detailed in the caption of Fig. 4. Simulation results reproduce experimental trends in Fig. 4A supporting the interpretation that the reaction kinetics is the factor limiting NP adsorption in experiments. Note that in simulations the typical adhesion timescales are much faster than the experimental ones. This is due to the simulation scheme employed that, as compared to experiments, drastically reduces the time between successive NP-LUV encounters, $\tau_{\text {diff }}$, while properly reproducing reaction times, $\tau_{\text {bond }}$, and the time taken by NPs to diffuse away from the interacting region, $\tau_{\text {coll }}$. This argument, along with the reasoning used above to justify the timescale of Fig. 4A, explains the similarity between simulation and experimental profiles that only differ by a time given by the ratio between the experimental and simulated $\tau_{\text {diff }}$.

To better clarify the role played by the reaction rates on adsorption kinetics, in Fig. 5A we visualize the time evolution of the number of adsorbed nanoparticles, calculated using different reaction rates. The latter are controlled by changing $k_{\text {on }}^{0, *}$ while keeping the ligand-receptor binding free energy, and therefore the thermodynamics of the system, constant. Consequently, the ligand-receptor off rate is also rescaled as $k_{\text {off }}^{i} \propto k_{\text {on }}^{0, *}($ Eq. 3$)$.

For $k_{\text {on }}^{0, *}, k_{\text {off }}^{i}=\infty$, the system experiences no kinetic hindrance from ligand-receptor reactions, and can therefore reach thermodynamic 
equilibrium. In these conditions, mobile receptors are observed to redistribute instantaneously among available NPs, progressively decreasing the average number of bonds per NP to stabilize a greater number of adsorbed nanoparticles, as displayed in Fig. 5B. In turn, Fig. 5A shows that for finite reaction rates the asymptotic number of adsorbed nanoparticles is much smaller than the one observed with $k_{\text {on }}^{0, *}=\infty$, implying that for $k_{\text {on }}^{0, *}<\infty$ the system fails to reach equilibrium. Indeed, even for the largest (finite) $k_{\text {on }}^{0, *}$ value we could test, $k_{\text {off }}^{i}$ becomes negligibly small $\left(\approx 10^{-9}\right.$ $\mathrm{s}^{-1}$ ) owing to the strong ligand-receptor affinity. Bond irreversibility results in the receptordepletion effect hypothesized above, where all adhering NPs quickly and irreversibly maximise the number of formed bonds, which in simulations is set to $N_{\text {bonds }}^{\max }=25$ (Fig. 5B). To enter a regime where adsorption is not limited by DNA denaturation, one would need $k_{\text {on }}^{0, *} \gtrsim \exp \left[-\beta \Delta G_{0}\right]$. Unfortunately, such values of $k_{\text {on }}^{0, *}$ are not computationally affordable. For $k_{\text {on }}^{0, *}=\infty$ bond formation occurs instantly upon NP-LUV collision, implying that initial aggregation is diffusion limited and occurs over typical timescales $\tau_{\text {diff }}$. Figure 5 shows that, at early times, the curve calculated for $k_{\text {on }}^{0, *}=10^{5}$ follows the same trend, indicating that in this regime the bond formation time $\tau_{\text {bonds }}$ is still significantly smaller than the collision time $\tau_{\text {coll }}$. The same is no longer true if $k_{\text {on }}^{0, *} \leq 10^{-1}$, for which the onset of NP adhesion is significantly delayed as the likelihood of forming a permanent NP-LUV bond upon collision is progressively reduced.

\section{Steady state}

From the time evolution of the fraction $f_{\mathrm{adh}}(t)$ of LUV-adhering NPs we can extract the asymptotic value $f_{\text {adh }}^{\text {ss }}$, corresponding to fraction of adhering nanoparticles at steady state (Eq. 8). In Fig. 6A, the experimental $f_{\mathrm{adh}}^{\mathrm{ss}}$ is show as a function of $R_{\mathrm{NP}: \mathrm{LUV}}$ and $\rho_{\mathrm{R}-\mathrm{LUV}}$ (full symbols), and compared to simulations results (empty symbols). We observe qualitative agreement between experiments and simula- tions, but the latter appear to underestimate $f_{\mathrm{adh}}^{\mathrm{ss}}$ in regimes of low $N_{\mathrm{R}-\mathrm{NP}}$. We ascribe this deviation to the fact that, in simulations, the average number of bonds $N_{\text {bonds }}^{\text {ss }}$ formed by each adhering NP at steady state is always maximised, and equal to $N_{\text {bonds }}^{\max }=25$ (Fig. 5). This is not always the case in experiments, as shown in Fig. $6 \mathrm{~B}$. $N_{\text {bonds }}^{\text {ss }}$ is estimated experimentally by assuming that $i$ ) all receptors are bound, and $i i$ ) receptors are evenly partitioned among adsorbed NPs. We observe that the experimental $N_{\text {bonds }}^{\text {ss }}$ is aways smaller than, or comparable to $N_{\text {bonds }}^{\max }$. More specifically $N_{\text {bonds }}^{\text {ss }}$ increases as a function of $\rho_{\mathrm{R}-\mathrm{LUV}}$ for $R_{\mathrm{NP}: \mathrm{LUV}}=70$, approaching $N_{\mathrm{bonds}}^{\max }$ for the highest tested receptor density, while remaining relatively small and constant for $R_{\mathrm{NP}: \mathrm{LUV}}=290$. Note that condition $i$ ) cannot be verified for $R_{\mathrm{NP}: \mathrm{LUV}}=7$, where $N_{\mathrm{R}-\mathrm{NP}}>N_{\text {bonds }}^{\max }$, thus the estimates for these samples are not included in Fig. 6. There are a number of effects that can result in $N_{\text {bonds }}^{\text {ss }}<N_{\text {bonds }}^{\max }$, not accounted for in simulations. First, in Fig. $6 \mathrm{~B}$ we assume that receptors are evenly distributed among all the adsorbed NPs, while it should not be excluded that those adhering at later stages may form less bonds than earlier nanoparticles. This nonideal behavior could emerge, for instance, as a consequence of non-selective (e.g. steric) interactions between ligands and receptors, that could significantly slow down bond formation and receptor sequestration by increasing $\tau_{\text {bond }}$, allowing more nanoparticles to bind the LUV. Additionally, $N_{\text {bonds }}^{\text {ss }}<N_{\text {bonds }}^{\max }$ may imply a degree of bond reversibility enabling receptor redistribution. This could be explained by an underestimation of the off rates, which in crowded environments may deviate sensibly from the theoretical predictions obtained in diluted conditions (Eq. 3). ${ }^{81}$ We point out once again that for $R_{\mathrm{NP}: \mathrm{LUV}}=290$ there is not enough room on the LUVs to accomodate all available nanoparticles, regardless on receptor availability. The geometrical limit to NP adsorption per LUV is $\sim 150$, corresponding to $f_{\text {adh }} \sim 0.5$. This value is similar to the asymptotic fractions experimentally observed for $R_{\mathrm{NP}: \mathrm{LUV}}=290$ in Fig. $6 \mathrm{~A}$. 


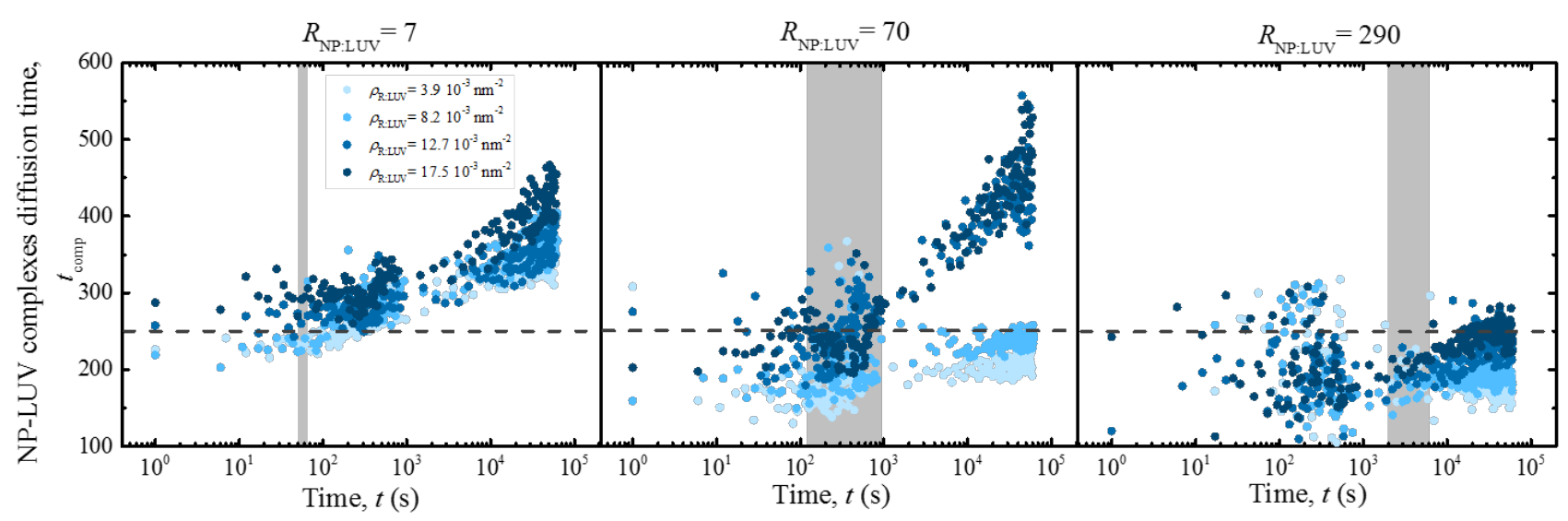

Figure 7: Diffusion time of NP-LUV complexes suggest LUV clustering. Decay time $\tau_{\text {comp }}$, describing the diffusivity of NP-LUV complexes shown as a function of the receptor surface density on LUVs $\rho_{\mathrm{R}-\mathrm{LUV}}$ for different NP:LUV concentration ratios $R_{\mathrm{NP}: \mathrm{LUV}}=7,70$ and 290 . The dashed lines represent the characteristic diffusion time of a $180 \mathrm{~nm}$ diameter liposome. The grey areas mark the range of adhesion times $\tau_{\text {adh }}$ measured for the different $\rho_{\mathrm{R}-\mathrm{LUV}}$ (Fig. 4 ).

\section{LUVs aggregation driven by NP bridging}

Further information on the evolution of the system can be gathered by monitoring the time dependence of the diffusion timescale $\tau_{\text {comp }}$ of the NP-LUV complexes (equation 7). As shown in Fig. 7, $\tau_{\text {comp }}$ exhibits different trends depending on $\rho_{\mathrm{R}-\mathrm{LUV}}$ (shown in different colors) and $R_{\mathrm{NP} \text { LUV }}$ (shown in different panels). In all cases $\tau_{\text {comp }}$ remains roughly constant for times shorter than the typical adhesion timescale $\tau_{\text {adh }}$, marked as a grey-shaded band spanning the adhesion timescales observed for different $\rho_{\mathrm{R}-\mathrm{LUV}}$. For $R_{\mathrm{NP}: \mathrm{LUV}}=7$, an increase of $\tau_{\text {adh }}$ above the value expected for an individual LUV is observed at later times, suggesting the possibility of NPs mediated LUV-LUV aggregation. The latter can occur because, after all NP are adsorbed, for $R_{\mathrm{NP}: \mathrm{LUV}}=7$ the system still features free receptors that can bind NPs on other LUVs, causing aggregation. Consistently, the same phenomenon is observed for samples with $R_{\mathrm{NP}: \mathrm{LUV}}=70$ and large receptor densities, which as demonstrated in Fig. 6A also reach a regime where NPs are depleted but receptors are still available. As expected, in samples with $R_{\mathrm{NP}: \mathrm{LUV}}=290$, LUVs remain monodisperse at all times, as the large excess of NPs is always sufficient to deplete all receptors and effectively passivate the LUV surface against NP bridging. Note that LUV aggregation is not expected to affect our ability to quantify the fraction of bound NPs, used to monitor adsorption kinetics: all NPs, whether adhering to an individual LUV or bridging two of them, would still be considered as bound. In turn, NP-mediated LUV bridging may have an effect on aggregation kinetics, as NPs can no longer be considered as "inactivated" after adhering to a LUV, and would still be able to sequestrate more receptors by forming LUV-NP-LUV bridges. However, in view of the faster diffusion kinetics of individual NPs compared to that of NP-LUV complexes, the adhesion of free NPs to LUVs is expected to dominate over the formation of aggregates, as long as free NPs remain available.

\section{Conclusions}

In summary, through a combination of dedicated experiments and coarse-grained simulations, we investigated the kinetics of adhesion of gold nanoparticles to artificial lipid vesicles mediated by near-irreversible ligand-receptor bonds. DNA strands grafted onto the surface of the nanoparticles and the liposomes play the role of both ligands and receptors. This model system is chosen to mimic interactions 
between nanoscale vectors and the surface of biological cells, a key process in several biological and medical scenarios, including cell invasion by viruses and parasites and cell targeting by means of synthetic nanomedical vectors. The rate of nanoparticle-vesicle adhesion is studied over a broad range of biologically and technologically relevant conditions, by varying the surface density of available membraneanchored receptors and the bulk concentration of nanoparticles. In both experiments and simulations, we find that adhesion rates are heavily suppressed in regimes where nanoparticles are in excess compared to available receptors. Observed trends are rationalized by comparing the timescales of individual kinetic processes at play, including the frequency and duration of nanoparticle-vesicle collisions and the typical time required to form a ligand-receptor bond. We determine that the observed slow kinetics arises from receptor depletion, as diffusive membrane-anchored receptors quickly segregate within the nanoparticle-vesicle adhesion region upon initial docking of individual nanoparticles. Nanoparticles adhering at early aggregation stages therefore sequestrate a significant proportion of the available receptors, reducing the rate of bond formation for latecoming nanoparticles and slowing down their adhesion. The near-irreversibility of ligandreceptor connections hinder bond redistribution, leading to kinetically trapped configurations in which the number of membraneadsorbed nanoparticles is not maximised.

Our observations have direct application in the design of medical nanovectors targeting cell membrane receptors: for a fixed receptor surface density, the adsorption rate of nanoscale probes can be maximised by improving bond reversibility or by reducing the number of connections that each probe can form, delaying receptor depletion. Besides biomedical relevance, our remarks can also inform the design of multivalent colloidal building blocks for the bottom-up production of advanced materials, an area in which the predictability of the equilibrium phase behaviors is still not matched by a similarly accurate control over relaxation kinetics. Our findings are also relevant to the modeling community. In particular, they highlight the importance of explicitly considering reaction rates when designing numerical simulations.

Acknowledgement RL, BMM, PC, LDM and GB acknowledge support from the WienerAnspach foundation. LDM acknowledges support from the Leverhulme Trust and the Isaac Newton Trust through an Early Career Fellowship (ECF-2015-494), and the Royal Society through a University Research Fellowship (UF160152). PC and LDM acknowledge support from the EPSRC Programme Grant CAPITALS (EP/J017566/1). PKJ and BMM are supported by the Fonds de la Recherche Scientifique de Belgique - FNRS under grant $\mathrm{n}^{\circ}$ MIS F.4534.17. Computational resources have been provided by the Consortium des Équipements de Calcul Intensif (CECI), funded by the Fonds de la Recherche Scientifique de Belgique - FNRS under grant $n^{\circ}$ 2.5020.11. RL acknowledge Simon Croegaert for the production of AuNPs and TEM images, Jonathan Goole from Pharmaceutics and Biopharmaceutics group (Université libre de Bruxelles) to give her access to Malvern Zetasizer NanoZS and Guy Vandenbussche from Structure and Function of Biological Membranes group (Université libre de Bruxelles) for letting her use Ultra Centrifuge.

\section{Supporting Information Avail- able}

The Supporting Information is available free of charge.

\section{References}

(1) Bidarimath, M.; Khalaj, K.; Kridli, R. T.; Kan, F. W. K.; Koti, M.; C., T. "Extracellular vesicle mediated intercellular communication at the porcine maternal-fetal interface: A new paradigm for conceptusendometrial cross-talk". Scientific Reports 2012, 7, 40476. 
(2) Tkach, M.; Théry, C. "Communication by Extracellular Vesicles: Where We Are and Where We Need to Go". Cell 2016, 164, 1226-1232.

(3) Turturici, G.; Tinnirello, R.; Sconzo, G.; Geraci, F. "Extracellular membrane vesicles as a mechanism of cell-to-cell communication: advantages and disadvantages". American Journal of PhysiologyCell Physiology 2014, 306, C621-C633, PMID: 24452373.

(4) Nolte-'t Hoen, E.; Cremer, T.; Gallo, R. C.; Margolis, L. B. "Extracellular vesicles and viruses: Are they close relatives?". Proceedings of the $\mathrm{Na}$ tional Academy of Sciences 2016, 113, 9155-9161.

(5) Rodrigues, M.; Fan, J.; Lyon, C.; Wan, M.; Y., H. "Role of Extracellular Vesicles in Viral and Bacterial Infections: Pathogenesis, Diagnostics, and Therapeutics". Theranostics 2018, 8 .

(6) Meckes, D. G.; Raab-Traub, N. "Microvesicles and Viral Infection". Journal of Virology 2011, 85, 12844-12854.

(7) Zhang, S.; Gao, H.; Bao, G. "Physical Principles of Nanoparticle Cellular Endocytosis". American Chemical Society Nano 2015, 9, 8655-8671, PMID: 26256227.

(8) Straubinger, R. M.; Hong, K.; Friend, D. S.; Papahadjopoulos, D. "Endocytosis of liposomes and intracellular fate of encapsulated molecules: Encounter with a low $\mathrm{pH}$ compartment after internalization in coated vesicles". Cell 1983, 32, 1069 - 1079.

(9) Ewers, H.; Helenius, A. "Lipid-Mediated Endocytosis". Cold Spring Harbor Perspectives in Biology 2011, 3.

(10) Hillaireau, H.; Couvreur, P. "Nanocarriers' entry into the cell: relevance to drug delivery". Cellular and Molecular Life Sciences 2009, 66, 2873-2896.
(11) Vasir, J. K.; Labhasetwar, V. "Quantification of the force of nanoparticle-cell membrane interactions and its influence on intracellular trafficking of nanoparticles". Biomaterials 2008, 29, 4244 - 4252.

(12) Lo, P. K.; Metera, K. L.; Sleiman, H. F. Self-assembly of three-dimensional DNA nanostructures and potential biological applications. Current opinion in chemical biology 2010, 14, 597-607.

(13) Varilly, P.; Angioletti-Uberti, S.; Mognetti, B.; Frenkel, D. "A general theory of DNA-mediated and other valence-limited colloidal interactions". The Journal of Chemical Physics 2012, 137, 094108-094122.

(14) Angioletti-Uberti, S.; Varilly, P.; Mognetti, B.; Tkachenko, A.; Frenkel, D. "Communication: A simple analytical formula for the free energy of ligandreceptor-mediated interactions". The Journal of Chemical Physics 2013, 138, 021102-021106.

(15) Parolini, L.; Mognetti, B. M.; Kotar, J.; Eiser, E.; Cicuta, P.; Di Michele, L. "Volume and porosity thermal regulation in lipid mesophases by coupling mobile ligands to soft membranes". Nature Communications 2015, 6, 5948.

(16) Shimobayashi, S. F.; Mognetti, B. M.; Parolini, L.; Orsi, D.; Cicuta, P.; Di Michele, L. "Direct measurement of DNA-mediated adhesion between lipid bilayers". Physical Chemistry Chemical Physics 2015, 17, 15615-15628.

(17) Di Michele, L.; Bachmann, S. J.; Parolini, L.; Mognetti, B. M. "Communication: Free energy of ligand-receptor systems forming multimeric complexes". The Journal of Chemical Physics 2016, 144, 161104.

(18) Angioletti-Uberti, S.; Mognetti, B. M.; Frenkel, D. "Theory and Simulation of 
DNA-Coated Colloids: a Guide for Rational Design". Physical Chemistry Chemical Physics 2016, 18, 6373-6393.

(19) Bachmann, S. J.; Kotar, J.; Parolini, L.; Saric, A.; Cicuta, P.; Di Michele, L.; Mognetti, B. M. "Melting transition in lipid vesicles functionalised by mobile DNA linkers". Soft Matter 2016, 12, 7804-7817.

(20) Bell, G.; Dembo, M.; Bongrand, P. Cell adhesion. Competition between nonspecific repulsion and specific bonding. Biophysical Journal 1984, 45, 1051 - 1064.

(21) Coombs, D.; Dembo, M.; Wofsy, C.; Goldstein, B. Equilibrium Thermodynamics of Cell-Cell Adhesion Mediated by Multiple Ligand-Receptor Pairs. Biophysical Journal 2004, 86, 1408 - 1423.

(22) Martinez-Veracoechea, F. J.; Frenkel, D. "Designing super selectivity in multivalent nano-particle binding". Proceedings of the National Academy of Sciences 2011, 108, 10963-10968.

(23) Rogers, W. B.; Shih, W. M.; Manoharan, V. N. "Using DNA to program the self-assembly of colloidal nanoparticles and microparticles". Nature Reviews $\mathrm{Ma}^{-}$ terials 2016, 1, 16008.

(24) Macfarlane, R. J.; O’Brien, M. N.; Petrosko, S. H.; Mirkin, C. A. "Nucleic acidmodified nanostructures as programmable atom equivalents: forging a new "table of elements" ". Angewandte Chemie - International Edition 2013, 52, 5688-5698.

(25) Jones, M. R.; Macfarlane, R. J.; Lee, B.; Zhang, J.; Young, K. L.; Senesi, A. J.; Mirkin, C. A. "DNA-nanoparticle superlattices formed from anisotropic building blocks". Nature materials 2010, 9, 913917.

(26) Jones, M. R.; Seeman, N. C.; Mirkin, C. A. "Programmable materials and the nature of the DNA bond". Science 2015, 347.
(27) Nykypanchuk, D.; Maye, M. M.; van der Lelie, D.; Gang, O. "DNA-guided crystallization of colloidal nanoparticles". Nature 2008, 451, 549-552.

(28) Chakraborty, I.; Meester, V.; van der Wel, C.; Kraft, D. J. Colloidal joints with designed motion range and tunable joint flexibility. Nanoscale 2017, 9, 7814-7821.

(29) Hong, S.; Leroueil, P. R.; Majoros, I. J.; Orr, B. G.; Baker, J. R.; Holl, M. M. B. "The Binding Avidity of a NanoparticleBased Multivalent Targeted Drug Delivery Platform". Chemistry and Biology 2007, 14, $107-115$.

(30) Mathai, M.; Ki, C. S.; M., W. G. "Polyvalent Interactions in Biological Systems: Implications for Design and Use of Multivalent Ligands and Inhibitors". Angewandte Chemie International Edition 37, 2754-2794.

(31) Cairo, C. W.; Gestwicki, J. E.; Kanai, M.; Kiessling, L. L. "Control of Multivalent Interactions by Binding Epitope Density". Journal of the American Chemical Society 2002, 124, 1615-1619, PMID: 11853434.

(32) Carlson, C. B.; Mowery, P.; Owen, R. M.; Dykhuizen, E. C.; Kiessling, L. L. "Selective Tumor Cell Targeting Using LowAffinity, Multivalent Interactions". American Chemical Society Chemical Biology 2007, 2, 119-127, PMID: 17291050.

(33) Chaudhuri, A.; Battaglia, G.; Golestanian, R. "The effect of interactions on the cellular uptake of nanoparticles". Physical Biology 2011, 8, 046002.

(34) Wibowo, A. S.; Singh, M.; Reeder, K. M.; Carter, J. J.; Kovach, A. R.; Meng, W.; Ratnam, M.; Zhang, F.; Dann, C. E. Structures of human folate receptors reveal biological trafficking states and diversity in folate and antifolate recognition. Proceedings of the National Academy of Sciences 2013, 110, 15180-15188. 
(35) Leunissen, M. E.; Dreyfus, R.; Cheong, F. C.; Grier, D. G.; Sha, R.; Seeman, N. C.; Chaikin, P. M. "Switchable self-protected attractions in DNAfunctionalized colloids". Nature Materials 2009, 8, $590 \mathrm{EP}$-.

(36) Leunissen, M. E.; Dreyfus, R.; Sha, R.; Seeman, N. C.; Chaikin, P. M. Quantitative Study of the Association Thermodynamics and Kinetics of DNA-Coated Particles for Different Functionalization Schemes. Journal of the American Chemical Society 2010, 132, 1903-1913.

(37) Di Michele, L.; Varrato, F.; Kotar, J.; Nathan, S. H.; Foffi, G.; Eiser, E. "Multistep kinetic self-assembly of DNA-coated colloids". Nature Communications 2013, 4, 1-7.

(38) Varrato, F.; Di Michele, L.; Belushkin, M.; Dorsaz, N.; Nathan, S. H.; Eiser, E.; Foffi, G. "Arrested demixing opens route to bigels". PNAS, USA 2012, 109, 1915519160.

(39) Parolini, L.; Kotar, J.; Di Michele, L.; Mognetti, B. M. "Controlling SelfAssembly Kinetics of DNA-Functionalized Liposomes Using Toehold Exchange Mechanism". American Chemical Society Nano 2016, 10, 2392-2398.

(40) Shenoy, V. B.; Freund, L. B. "Growth and shape stability of a biological membrane adhesion complex in the diffusionmediated regime". Proceedings of the $\mathrm{Na}$ tional Academy of Sciences 2005, 102, 3213-3218.

(41) Atilgan, E.; Ovryn, B. Nucleation and growth of integrin adhesions. Biophysical Journal 2009, 96, 3555-3572.

(42) Rogers, W. B.; Sinno, T.; Crocker, J. C. Kinetics and non-exponential binding of DNA-coated colloids. Soft Matter 2013, 9, 6412-6417.

(43) Licata, N. A.; Tkachenko, A. V. Kinetic limitations of cooperativity-based drug delivery systems. Physical review letters 2008, 100, 158102.

(44) Xu, Q.; Feng, L.; Sha, R.; Seeman, N.; Chaikin, P. Subdiffusion of a sticky particle on a surface. Physical review letters 2011, 106, 228102.

(45) Eze, N. A.; Sullivan, R. S.; Milam, V. T. Analysis of in Situ LNA and DNA Hybridization Events on Microspheres. Biomacromolecules 2017, 18, 1086-1096.

(46) Li, M.-H.; Choi, S. K.; Leroueil, P. R.; Baker, J. R. Evaluating Binding Avidities of Populations of Heterogeneous Multivalent Ligand-Functionalized Nanoparticles. ACS Nano 2014, 8, 5600-5609, PMID: 24810868.

(47) Li, M.-H.; Zong, H.; Leroueil, P. R.; Choi, S. K.; Baker, J. R. Ligand Characteristics Important to Avidity Interactions of Multivalent Nanoparticles. Bioconjugate Chemistry 2017, 28, 16491657, PMID: 28398751.

(48) Beales, P. A.; Vanderlick, T. K. Specific binding of different vesicle populations by the hybridization of membrane-anchored DNA. The Journal of Physical Chemistry A 2007, 111, 12372-12380.

(49) Hadorn, M.; Hotz, P. E. DNA-mediated self-assembly of artificial vesicles. PLoS One 2010, 5, e9886.

(50) Beales, P. A.; Nam, J.; Vanderlick, T. K. Specific adhesion between DNA-functionalized 'Janus' vesicles: size-limited clusters. Soft Matter 2011, 7, 1747-1755.

(51) Hadorn, M.; Boenzli, E.; Sørensen, K. T.; De Lucrezia, D.; Hanczyc, M. M.; Yomo, T. Defined DNA-mediated assemblies of gene-expressing giant unilamellar vesicles. Langmuir 2013, 29, 1530915319 .

(52) Pontani, L.-L.; Jorjadze, I.; Viasnoff, V.; Brujic, J. Biomimetic emulsions reveal 
the effect of mechanical forces on cellcell adhesion. Proceedings of the National Academy of Sciences 2012, 109, 98399844.

(53) Feng, L.; Pontani, L.-L.; Dreyfus, R.; Chaikin, P.; Brujic, J. Specificity, flexibility and valence of DNA bonds guide emulsion architecture. Soft Matter 2013, 9, 9816-9823.

(54) Quinn, P.; Griffiths, G.; GRAHAM Warren, G. "Density of newly synthesized plasma membrane proteins in intracellular membranes II. Biochemical studies". The Journal of Cell Biology 1984, 98, 2142-7.

(55) Lukeš, T.; Glatzová, D.; Kvíčalová, Z.; Levet, F.; Benda, A.; Letschert, S.; Sauer, M.; Brdička, T.; Lasser, T.; Cebecauer, M. "Quantifying protein densities on cell membranes using super-resolution optical fluctuation imaging". Nature Communications 2017, 8 .

(56) Banchelli, M.; Betti, F.; Berti, D.; Caminati, G.; Bombelli, F. B.; Brown, T.; Wilhelmsson, L. M.; Nordén, B.; Baglioni, P. "Phospholipid Membranes Decorated by Cholesterol-Based Oligonucleotides as Soft Hybrid Nanostructures". The Journal of Physical Chemistry $B$ 2008, 112, 10942-10952, PMID: 18693696.

(57) Bunge, A.; Loew, M.; Pescador, P.; Arbuzova, A.; Brodersen, N.; Kang, J.; Dähne, L.; Liebscher, J.; Herrmann, A.; Stengel, G.; Huster, D. "Lipid Membranes Carrying Lipophilic Cholesterol-Based Oligonucleotides-Characterization and Application on Layer-by-Layer Coated Particles". The Journal of Physical Chemistry B 2009, 113, 16425-16434, PMID: 19957915.

(58) Czogalla, A.; Franquelim, H. G.; Schwille, P. DNA Nanostructures on Membranes as Tools for Synthetic Biology. Biophysical Journal 2016, 110, 1698 $-1707$.
(59) Kimling, J.; Maier, M.; Okenve, B.; Kotaidis, V.; Ballot, H.; Plech, A. "Turkevich Method for Gold Nanoparticle Synthesis Revisited". The Journal of Physical Chemistry $B$ 2006, 110, 15700-15707, PMID: 16898714 .

(60) Mirkin, C.; Letsinger, R.; Mucic, R.; Storhoff, J. "A DNA-based method for rationally assembling nanoparticles into macroscopic materials". Nature 1996, 382, 607-609.

(61) SantaLucia, J. "A unified view of polymer, dumbbell, and oligonucleotide DNA nearest-neighbor thermodynamics". Proceedings of the National Academy of Sciences 1998, 95, 1460.

(62) Myers, B. D.; Lin, Q.-Y.; Wu, H.; Luijten, E.; Mirkin, C. A.; Dravid, V. P. Size-Selective Nanoparticle Assembly on Substrates by DNA Density Patterning. ACS Nano 2016, 10, 5679-5686, PMID: 27192324.

(63) Kanayama, N.; Sekine, T.; Ozasa, K.; Kishi, S.; Nyu, T.; Hayashi, T.; Maeda, M. Terminal-Specific Interaction between Double-Stranded DNA Layers: Colloidal Dispersion Behavior and Surface Force. Langmuir 2016, 32, 13296-13304, PMID: 27951695.

(64) Kim, Y.; Macfarlane, R. J.; Jones, M. R.; Mirkin, C. A. Transmutable nanoparticles with reconfigurable surface ligands. Science 2016, 351, 579-582.

(65) Doyen, M.; Bartik, K.; Bruylants, G. DNA-Promoted Auto-Assembly of Gold Nanoparticles: Effect of the DNA Sequence on the Stability of the Assemblies. Polymers 2013, 5, 1041-1055.

(66) Zhang, Y.; Zhou, H.; Ou-Yang, Z.-C. "Stretching Single-Stranded DNA: Interplay of Electrostatic, Base-Pairing, and Base-Pair Stacking Interactions". Biophysical Journal 2001, 81, 1133 - 1143. 
(67) Bachmann, S. J.; Petitzon, M.; Mognetti, B. M. "Bond formation kinetics affects self-assembly directed by ligand-receptor interactions". Soft Matter 2016, 12, 9585-9592.

(68) Zhdanov, V. P. Multivalent ligandreceptor-mediated interaction of small filled vesicles with a cellular membrane. Phys. Rev. E 2017, 96, 012408.

(69) Dasgupta, S.; Auth, T.; Gompper, G. Shape and orientation matter for the cellular uptake of nonspherical particles. Nano letters 2014, 14, 687-693.

(70) Tzlil, S.; Deserno, M.; Gelbart, W. M.; Ben-Shaul, A. A statisticalthermodynamic model of viral budding. Biophysical journal 2004, 86, 2037-2048.

(71) Schubertová, V.; MartinezVeracoechea, F. J.; Vácha, R. Influence of ligand distribution on uptake efficiency. Soft Matter 2015, 11, 2726-2730.

(72) Di Michele, L.; Jana, P. K.; Mognetti, B. M. Steric interactions between mobile ligands facilitate complete wrapping in passive endocytosis. Phys. Rev. E 2018, 98, 032406.

(73) Angioletti-Uberti, S. Theory, simulations and the design of functionalized nanoparticles for biomedical applications: A Soft Matter Perspective. npj Computational Materials 2017, 3, 48.

(74) Šarić, A.; Cacciuto, A. Fluid Membranes Can Drive Linear Aggregation of Adsorbed Spherical Nanoparticles. Phys. Rev. Lett. 2012, 108, 118101.

(75) Parveen, N.; Rimkute, I.; Block, S.; Rydell, G. E.; Midtvedt, D.; Larson, G.; Hytönen, V. P.; Zhdanov, V. P.; Lundgren, A.; Höök, F. Membrane Deformation Induces Clustering of Norovirus Bound to Glycosphingolipids in a Supported CellMembrane Mimic. The Journal of Physical Chemistry Letters 2018, 9, 2278-2284, PMID: 29624391.
(76) Gillespie, D. T. "Exact stochastic simulation of coupled chemical reactions". The Journal of Physical Chemistry 1977, 81, 2340-2361.

(77) Berne, B. J.; Pecora, R. "Dynamic Light Scattering with applications to Chemistry, Biology, and Physics"; Dover Publications. INC., 2000.

(78) Vasina, E. N.; Déjardin, P. Kinetics of Adsorption, Desorption, and Exchange of $\alpha$-Chymotrypsin and Lysozyme on Poly(ethyleneterephthalate) Tracked Film and Track-Etched Membrane. Biomacromolecules 2003, 4, 304-313, PMID: 12625725 .

(79) Douglas, J. F.; Johnson, H. E.; Granick, S. A Simple Kinetic Model of Polymer Adsorption and Desorption. Science 1993, 262, 2010-2012.

(80) Jia, L.; Lai, P. Kinetics and structure of irreversibly adsorbed polymer layers. The Journal of Chemical Physics 1996, 105, 11319-11325.

(81) Paramanathan, T.; Reeves, D.; Friedman, L. J.; Kondev, J.; Gelles, J. A general mechanism for competitor-induced dissociation of molecular complexes. $\mathrm{Na}$ ture Communications 2014, 5, 5207. 
Graphical TOC Entry

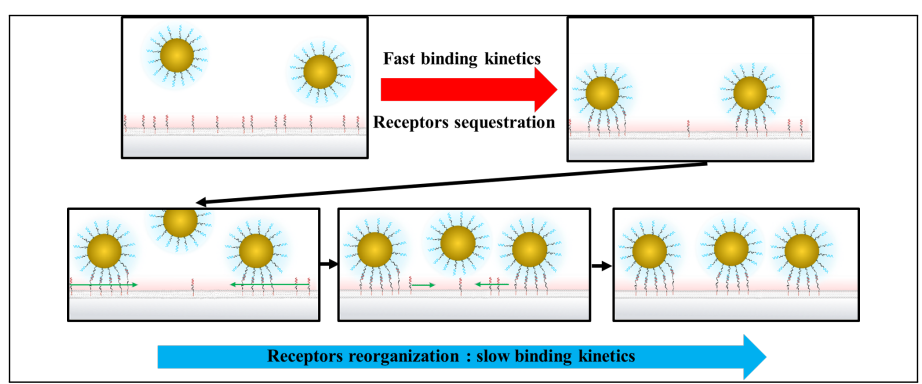

